\title{
Emergence of patterns in driven and in autonomous spatiotemporal systems
}

\author{
M. G. Cosenza, M. Pineda and A. Parravano \\ Centro de Astrofísica Teórica, Facultad de Ciencias Universidad de Los Andes, \\ Mérida, Apartado Postal 26, Mérida 5251, Venezuela.
}

(Dated: )

\begin{abstract}
The relationship between a driven extended system and an autonomous spatiotemporal system is investigated in the context of coupled map lattice models. Specifically, a locally coupled map lattice subjected to an external drive is compared to a coupled map system with similar local couplings plus a global interaction. It is shown that, under some conditions, the emergent patterns in both systems are analogous. Based on the knowledge of the dynamical responses of the driven lattice, we present a method that allows the prediction of parameter values for the emergence of ordered spatiotemporal patterns in a class of coupled map systems having local coupling and general forms of global interactions.

PACS numbers: 05.45.-a, 89.75.Kd
\end{abstract}

The phenomenon of pattern formation induced by external forcing on spatiotemporal systems, such as chemical reactions [1, 2, 3] or granular media [4, 5, 6], have received much attention. Similarly, there has been recent interest in experimental investigations of spontaneous pattern formation and emergence of collective behaviors in spatially extended systems of interacting dynamical elements, such as one-dimensional arrays of electrochemical oscillators [7], chemical and hydrodynamical systems with global coupling [8, 9], and populations of chaotic electrochemical cells having both local and global interactions [10]. In this context, coupled map lattices have provided fruitful theoretical models for studying and predicting a variety of dynamical processes, including pattern formation, in spatiotemporal chaotic systems possessing different kinds of interaction topologies, such as local couplings, regular geometries, inhomogeneous and disordered networks, and global coupling [11].

In this article we investigate the relationship between forced spatiotemporal systems and autonomous dynamical systems possessing both local and global interactions in the framework of coupled map lattices. We analyze the emergence of ordered patterns in forced spatiotemporal systems by using a model of a coupled map lattice subjected to an external drive. We show that, under some circumstances, this system is analogous to an autonomous coupled map system with both local and global interactions. Our approach is motivated by the observation that a globally coupled map system behaves similarly to a single map subjected to an external drive and that this analogy may be used to describe the formation of dynamical clusters in globally coupled maps 12].

As a model of an autonomous spatiotemporal system, we consider the following coupled map network possessing both, local and global interactions [13],

$$
x_{t+1}^{i}=\left(1-\epsilon_{2}\right) f\left(x_{t}^{i}\right)+\frac{\epsilon_{1}}{2}\left[\left(f\left(x_{t}^{i+1}\right)+f\left(x_{t}^{i-1}\right)-2 f\left(x_{t}^{i}\right)\right]+\epsilon_{2} H\left(x_{t}^{1}, x_{t}^{2}, x_{t}^{3}, \ldots, x_{t}^{N}\right),\right.
$$

which can be compared to a one-dimensional coupled map lattice subjected to a uniform external drive,

$$
s_{t+1}^{i}=\left(1-\epsilon_{2}\right) f\left(s_{t}^{i}\right)+\frac{\epsilon_{1}}{2}\left[\left(f\left(s_{t}^{i+1}\right)+f\left(s_{t}^{i-1}\right)-2 f\left(s_{t}^{i}\right)\right]+\epsilon_{2} L_{t} .\right.
$$

In Eq. (11),$x_{t}(i)$ gives the state of element $i(i=1,2, \ldots, N)$ at discrete time $t ; N$ is the size of the system; $f(x)$ describes the (nonlinear) local dynamics; $\epsilon_{1}$ and $\epsilon_{2}$ are the local and global coupling parameters, respectively; and $H\left(x_{t}^{1}, x_{t}^{2}, x_{t}^{3}, \ldots, x_{t}^{N}\right)$ is a global coupling function of $N$ variables assumed to be invariant to argument permutations; that is $H\left(\ldots, x_{t}^{i}, \ldots, x_{t}^{j}, \ldots\right)=H\left(\ldots, x_{t}^{j}, \ldots, x_{t}^{i}, \ldots\right) ; \forall i, j$. This property of the global coupling function ensures that, at any time, each element of the lattice is subjected to the same influence of the global coupling term. Thus the interaction $H$ provides a global feedback. As a specific example, we shall take the usual mean field global coupling $H=\frac{1}{N} \sum_{i=1}^{N} f\left(x_{t}^{i}\right)$; although the analysis presented here is applicable whenever a permutable coupling function of $N$ variables appears in the autonomous spatiotemporal dynamical system Eq. (11). In Eq. (2), $s_{t}^{i}$ is the state of element $i$ in the driven lattice, $f\left(s_{t}^{i}\right)$ is the same local dynamics as in Eq. (1), $\epsilon_{1}$ measures the local coupling, $\epsilon_{2}$ represents the coupling strength to the external forcing, and $L_{t}$ is the uniform driving term. In general, $L_{t}$ may be any function of time. We assume periodic boundary conditions and a quadratic map $f(x)=1-r x^{2}$ as local dynamics in both Eq. (1) and Eq. (2).

The analogy between an autonomous coupled map system having both local and global interactions and a uniformly driven lattice arises because in the former system (Eq.1) all the elements in the network are affected by the global coupling in exactly the same way at any time, and therefore the behavior of any map $x_{t}^{i}$ in Eq.(11) is equivalent to the behavior of any element in the driven lattice (Eq. 2) with $L_{t}=H$ and initial conditions $s_{o}^{i}=x_{o}^{i}$. Additionally, if 
the autonomous coupled map system in Eq.(1) reaches an ordered spatiotemporal pattern, its corresponding global coupling function $H$ follows an ordered motion. Thus the associated driven lattice, Eq. (2), with a periodic forcing $L_{t}$ should exhibit an ordered spatial pattern similar to that of the coupled map system having both local and global interactions. In particular, periodic drives resulting in periodic patterns in the driven lattice Eq.(2) may be employed to predict the emergence of periodic patterns in autonomous systems described by Eq.(1), regardless of the specific functional form of the permutable global coupling $H$ and without doing direct simulations on the autonomous system.

Let us analyze the dynamical response of the driven coupled map lattice subjected to periodic forcing. For a drive having period $T$, we denote the sequence of $T$ values adopted by $L_{t}$ as $\left\{L_{1}, L_{2}, \ldots, L_{T}\right\}$. For some parameter values and initial conditions, the resulting dynamics of the driven system (Eq.2) may reach an ordered spatiotemporal pattern corresponding to lattice configurations that are periodic in both, space and time. The spatial wavelength $k$ and the temporal period $p$ of the dynamics of the elements are defined by the relations $s_{t}^{i}=s_{t}^{i+k}$ and $s_{t}^{i}=s_{t+p}^{i}$, respectively. In these cases it suffices to consider only the dynamics of the $k$ coupled elements forming a wavelength, and therefore the behavior of driven lattice (Eq.2) can be analyzed by considering a reduced driven system of $k$ coupled maps.

An ordered spatiotemporal pattern having wavelength $k$ and period $p$ emerging in a periodically driven lattice can be characterized by the $k \times p$ matrix

$$
S(k, p)=\left(\begin{array}{ccc}
\sigma_{1}^{1} & \ldots & \sigma_{1}^{k} \\
\vdots & \ddots & \vdots \\
\sigma_{p}^{1} & \ldots & \sigma_{p}^{k}
\end{array}\right)
$$

where the $m$ th column contains the consecutive $p$ values of the periodic response of an element $m$ belonging to a spatial wavelength, $(m=1,2, \ldots, k)$; and the $n$th row displays the values of all the $k$ elements in a wavelength (a snapshot) at the cyclic time step $n,(n=1,2, \ldots, p)$. The driven lattice may reach different asymptotic ordered spatiotemporal patterns $S(k, p)$ depending on the initial conditions; i.e. multistability is possible.

Once a pattern $S(k, p)$ appears in the driven lattice for some values of the parameters, it must satisfy the following set of $k \times p$ nonlinear algebraic equations,

$$
\sigma_{n+1}^{m}=\left(1-\epsilon_{1}-\epsilon_{2}\right) f\left(\sigma_{n}^{m}\right)+\frac{\epsilon_{1}}{2}\left[\left(f\left(\sigma_{n}^{m+1}\right)+f\left(\sigma_{n}^{m-1}\right)\right]+\epsilon_{2} L_{n} .\right.
$$

In general, for emergent periodic patterns the ratio $p / T=\nu$ is a natural number that characterizes the resonance between the period of the driving term and the resulting period of the lattice. Thus, the sequence of values $\left\{L_{1}, L_{2}, \ldots, L_{T}\right\}$ repeat themselves $\nu$ times in the right hand side of Eqs.(四). In addition, the presence of symmetries in a spatiotemporal pattern may reduce the number of independent variables in the above set of equations. Eqs. (A) may yield several sets of solutions for the orbits $\sigma_{n}^{m}$, however only the stable ones will be observed as asymptotic patterns in the driven lattice. As examples, consider the following patterns in the driven lattice, characterized by the given matrix and satisfying the indicated relations, corresponding to Eqs.(4):

(1) Frozen $(p=1)$ wavelength $k=2$, with constant drive $L_{t}=\left\{L_{1}\right\}$,

$$
\begin{gathered}
S(2,1)=(a b) \\
a=\left(1-\epsilon_{1}-\epsilon_{2}\right) f(a)+\epsilon_{1} f(b)+\epsilon_{2} L_{1} \\
b=\left(1-\epsilon_{1}-\epsilon_{2}\right) f(b)+\epsilon_{1} f(a)+\epsilon_{2} L_{1} .
\end{gathered}
$$

(2) Out of phase, wavelength $k=2$ and period $p=2$, with constant drive $\left\{L_{1}\right\}$,

$$
\begin{gathered}
S(2,2)=\left(\begin{array}{ll}
a & b \\
b & a
\end{array}\right) ; \\
a=\left(1-\epsilon_{1}-\epsilon_{2}\right) f(b)+\epsilon_{1} f(a)+\epsilon_{2} L_{1} \\
b=\left(1-\epsilon_{1}-\epsilon_{2}\right) f(a)+\epsilon_{1} f(b)+\epsilon_{2} L_{1} .
\end{gathered}
$$

(3) Wavelength $k=2$ and period $p=4$, with period-two drive $\left\{L_{1}, L_{2}\right\}$,

$$
S(2,4)=\left(\begin{array}{ll}
a & b \\
c & d \\
b & a \\
d & c
\end{array}\right)
$$




$$
\begin{aligned}
& c=\left(1-\epsilon_{1}-\epsilon_{2}\right) f(a)+\epsilon_{1} f(b)+\epsilon_{2} L_{1} \\
& d=\left(1-\epsilon_{1}-\epsilon_{2}\right) f(b)+\epsilon_{1} f(a)+\epsilon_{2} L_{1} \\
& a=\left(1-\epsilon_{1}-\epsilon_{2}\right) f(d)+\epsilon_{1} f(c)+\epsilon_{2} L_{2} \\
& b=\left(1-\epsilon_{1}-\epsilon_{2}\right) f(c)+\epsilon_{1} f(d)+\epsilon_{2} L_{2} .
\end{aligned}
$$

(4) Wavelength $k=3$ and period $p=3$, with constant drive $\left\{L_{1}\right\}$,

$$
\begin{gathered}
S(3,3)=\left(\begin{array}{ccc}
a & b & c \\
b & c & a \\
c & a & b
\end{array}\right) ; \\
a=\left(1-\epsilon_{1}-\epsilon_{2}\right) f(c)+\frac{\epsilon_{1}}{2}[f(b)+f(a)]+\epsilon_{2} L_{1} \\
b=\left(1-\epsilon_{1}-\epsilon_{2}\right) f(a)+\frac{\epsilon_{1}}{2}[f(c)+f(b)]+\epsilon_{2} L_{1} \\
c=\left(1-\epsilon_{1}-\epsilon_{2}\right) f(b)+\frac{\epsilon_{1}}{2}[f(a)+f(c)]+\epsilon_{2} L_{1} .
\end{gathered}
$$

For the above examples, the orbits $a, b, c, d$ can be obtained as functions of the parameters $\epsilon_{1}, \epsilon_{2}, r$, and the values $L_{1}$ and $L_{2}$.

In practice, if we are searching for the orbits $\sigma_{n}^{m}$ in a specific stable pattern $S(k, p)$, we can construct a bifurcation diagram of a periodically driven lattice of $N$ coupled maps ( $N$ multiple of $k$ ) as a function of some parameter of the system, and look for windows where that pattern appears in the diagram. This may require exploring for appropriate initial conditions. Figures 1(a)-(d) show bifurcation diagrams of driven lattices as a function of either $\epsilon_{1}$ or $\epsilon_{2}$, where the patterns given in examples (1)-(4), additionally to other spatiotemporal states, arise. Fig. 1(a) shows the bifurcation diagram of a lattice subjected to a constant drive $L_{1}=0.39$ as a function of the coupling parameter of the drive $\epsilon_{2}$, with fixed local coupling $\epsilon_{1}=0.54$. As $\epsilon_{2}$ is varied, the steadily driven system exhibits several spatiotemporal states, such as synchronization (both chaotic and periodic), a pattern $S(2,1)$, and quasiperiodic $k=2$ wavelength. The stationary orbits $a$ and $b$ (for either the odd or even elements of the lattice) in the pattern $S(2,1)$ arise in the bifurcation diagram of Fig. 1(a) as functions of the parameter $\epsilon_{2}$. The values of the instantaneous mean field of the wavelength in each pattern $S(k, p)$, given by $\langle f\rangle_{n}=\frac{1}{k} \sum_{m=1}^{k} f\left(\sigma_{n}^{m}\right)$, are also plotted on the corresponding regions of the bifurcation diagrams. In the case of Fig. 1(a), we get $\langle f\rangle=[f(a)+f(b)] / 2$, independent of time, on the region $S(2,1)$. Note that at $\epsilon_{2}=0.56$, the mean field $\langle f\rangle$ is equal to the value of the drive $L_{1}=0.39$ in Fig.1(a). Since $\langle f\rangle$ also corresponds to the mean field of the entire lattice, it may be inferred that for the values of the parameters $r=2, \epsilon_{1}=0.54$, and $\epsilon_{2}=0.56$, an autonomous coupled system described by Eq. (11) with mean field global coupling may also exhibit a spatiotemporal frozen pattern with $k=2$ and $p=1$, while sustaining a constant value of its global coupling function at $H=0.39$. For those parameter values, the driven lattice Eq. (2) and the autonomous system Eq. (1) are equivalent. Note that this prediction is being made without direct numerical simulation on the autonomous spatiotemporal system. Moreover, the intersection of $\langle f\rangle$ with the constant value of $L_{1}$ in the diagram of the driven lattice in Fig. 1(a) readily provides a set of initial conditions for the odd and even elements $x_{t}^{i}$ in Eq. (1) for observing the spatiotemporal pattern $S(2,1)$ in the autonomous coupled system at the parameter values $\epsilon_{1}=054$, $\epsilon_{2}=0.56$, and $r=2$; that is, $x_{t}^{i}=a=0.017$, for $i$ even; and $x_{t}^{i}=b=0.776$, for $i$ odd. Similar predictions of parameter values and initial conditions for the emergence of the spatiotemporal patterns $S(2,2), S(2,4)$, and $S(3,3)$ of examples (2)-(4) in the autonomous system Eq.(1) can be made from the intersections of the curves $\langle f\rangle_{n}$ with the values of $L_{t}$ in the diagrams of the periodically driven lattices of Fig. 1(b), 1(c) and 1(d), respectively. Figs. 2(a)-2(d) display the spatiotemporal patterns $S(k, p)$ of examples (1)-(4) emerging in the autonomous system Eq.(11) while sustaining either a constant or a periodic mean field coupling function, at parameter values and orbits predicted from the analogy with the driven coupled map lattice.

These results suggest that a complete equivalence between a lattice driven with period $T$ (Eq.2), that gives rise to a spatiotemporal pattern $S(k, p)(p / T=\nu)$, and an autonomous system (Eq.11) can be established when the following conditions are satisfied

$$
L_{n}=H(\ldots, \underbrace{\sigma_{n}^{1}, \sigma_{n}^{2}, \ldots, \sigma_{n}^{k}}_{N / k \text { times }}, \ldots)
$$

where $n=1, \ldots, T$; and where the arguments of $H$ are the $k$ elements in the $n$th row of the matrix $S(k, p)$, repeated $N / k$ times. For convenience, we denote the right hand side of Eqs. (13) by $H\left(\sigma_{n}^{m}\right)$. For given parameters $r, \epsilon_{1}$, and $\epsilon_{2}$, the orbits $\sigma_{n}^{m}$ of the driven lattice depend on the periodic drive $L_{t}=\left\{L_{1}, L_{2}, \ldots, L_{T}\right\}$, according to Eq. (㺼). Thus, Eqs. (13) constitute a set of $T$ nonlinear equations for the values $\left\{L_{1}, L_{2}, \ldots, L_{T}\right\}$. The solutions $L_{t}^{*}=\left\{L_{1}^{*}, L_{2}^{*}, \ldots, L_{T}^{*}\right\}$ of Eqs. (13) predict that the autonomous system (Eq.11) with local and global interactions possesses a state characterized by the spatiotemporal pattern $S(k, p)$ with orbits $x_{n}^{m}=\sigma_{n}^{m}\left(L_{t}^{*}\right)$, and by the periodic global coupling function motion 
$H=L_{1}^{*}, H=L_{2}^{*}, \ldots, H=L_{T}^{*}$. As an illustration, consider the pattern $S(2,1)$ whose orbits $a\left(r, \epsilon_{1}, \epsilon_{2}, L_{1}\right)$ and $b\left(r, \epsilon_{1}, \epsilon_{2}, L_{1}\right)$ can be obtained from Eqs. (6). Then Eqs. (13) reduce to just one equation, which in the case of a mean field coupling function $H=\sum_{i}^{N} f\left(x_{t}^{i}\right)$, yields the solution

$$
L_{1}^{*}=\frac{1}{2 r\left(\epsilon_{2}+2 \epsilon_{1}-1\right)} .
$$

Thus for the set of parameters satisfying the above relation, the pattern $S(2,1)$ can emerge in both, a steadily driven lattice with $L_{t}=\left\{L_{1}^{*}\right\}$ and an autonomous system sustaining a constant mean field coupling $H=L_{1}^{*}$.

Fig. 3 displays the function $H\left(\sigma_{n}^{m}\right)$ corresponding to mean field coupling as a function of the constant drive $L_{1}$ for the patterns of examples (1), (2) and (4). The intersections of the curves with the diagonal give the solutions $L_{1}^{*}$ to Eq. (13) for each pattern indicated. Note that Eqs. (13) can be used to predict if a given pattern $S(k, p)$ observed in the driven lattice may emerge in diverse autonomous systems possessing different functional forms of the global coupling function $H$. For instance, one may ask if the pattern $S(2,2)$ of example (2) can also exist in an autonomous system Eq. (1) with a constant value of a global coupling function given by the geometric mean, $H=\prod_{i}^{N}\left|x_{t}^{i}\right|^{1 / N}$. Fig. 3 shows $H\left(\sigma_{n}^{m}\right)$ for this form of global coupling associated to the pattern $S(2,2)$ (slash-dotted curve) as a function of $L_{1}$, giving a solution $L_{1}^{*}=H=0.3418$ at the intersection with the diagonal. This prediction has been verified for this autonomous system.

In summary, we have studied the emergence of induced ordered patterns in forced spatiotemporal systems by using a model of a one-dimensional coupled map lattice subjected to an external drive. Under some circumstances, this system is analogous to an autonomous coupled map system possessing a similar local coupling and an additional global interaction that acts as a global feedback. Thus, by exploring the dynamical responses of a driven spatiotemporal system, one can get insight into the conditions for the emergence of specific patterns in a class of autonomous spatiotemporal systems. Once an ordered pattern $S(k, p)$ appears in a driven lattice for some values of the parameters, the same pattern is expected to spontaneously arise in a family of related autonomous coupled map systems that satisfy Eqs. (13), which constitute the link between both systems. Conversely, a pattern observed in an autonomous system with both local and global interactions should also exist in an associated driven system with similar local couplings for some appropriate period of the external drive. This equivalence could be tested in experimental settings of interacting dynamical elements [7, 8, 9, 10. The method has been applied to some simple cases; however it can be used for more complicated patterns. Although we have considered one-dimensional diffusive local couplings, the analogy between a uniform external drive and a global interaction can be applied to any network of coupled maps. The relation between extended systems subjected to non-uniform driving fields and spatiotemporal autonomous systems is an interesting problem for future research.

This work was supported by Consejo de Desarrollo Científico, Humanístico y Tecnológico of Universidad de Los Andes, Mérida, Venezuela.

[1] A. L. Lin, A. Hagberg, A Ardalea, H. Bertram, H. L. Swinney, and E. Meron, Phys. Rev. E 62, 3790 (2000).

[2] W, Wang, I. Z. Kiss, and J. L. Hudson, Phys. Rev. Lett. 86, 4954 (2001).

[3] C. Hemming and R. Kapral, Physica A 306, 199 (2002).

[4] P. B. Umbanhowar, F. Melo, and H. L. Swinney, Nature (London) 382, 793 (1996).

[5] P. B. Umbanhowar, F. Melo, and H. L. Swinney, Physica A 249, 1 (1998).

[6] T. Metcalf, J. B. Knight, and H. M. Jaeger, Physica A 236, 202 (1997).

[7] W. Wang, I. Z. Kiss, and J. L. Hudson, J. Phys. Chem. B 1032178 (1999).

[8] W, Wang, I. Z. Kiss, and J. L. Hudson, Chaos 10248 (2000).

[9] K. Miyakawa and K. Yamada, Physica D, 151, 217 (2001).

[10] I. Z. Kiss, Y. Zhai, and J. L. Hudson, Phys. Rev. Lett. 88, 238301 (2002).

[11] Chaos 2, No. 3 (1992), focus issue on Coupled Map Lattices; edited by K.Kaneko.

[12] A. Parravano and M. G. Cosenza, Phys. Rev. E 58, 1665 (1998).

[13] N. B. Ouchi and K. Kaneko, Chaos 10, 359 (2000). 

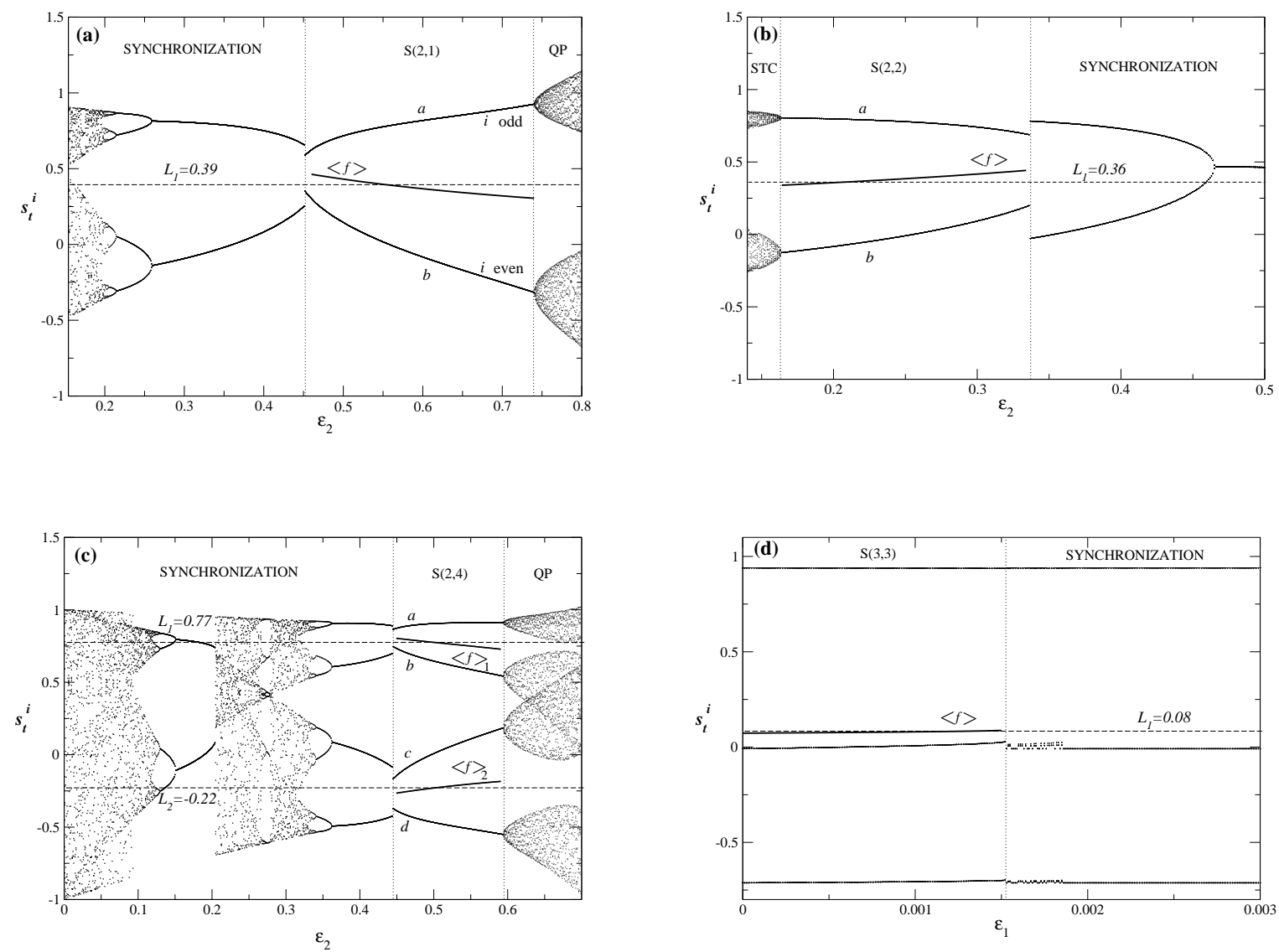

FIG. 1: Bifurcation diagrams of the orbits $s_{t}^{i}$ of the driven lattice (Eq.2) with size $N=30$ and fixed local parameter $r=2$. The values of the periodic drive $L_{t}$ are shown with dashed lines. Orbits $a, b, c, d$, corresponding to patterns $S(k, p)$ of examples (1)-(4) are indicated. The mean $\langle f\rangle_{n}$ is drawn with thick lines on each region where a pattern $S(k, p)$ appears. Regions where synchronization occurs are identified; regions of quasiperiodic behavior are labelled QP, and those of spatiotemporal chaos are labelled STC. (a) $\epsilon_{1}=0.54$, and constant drive $L_{1}=0.39$; bifurcation parameter is $\epsilon_{2}$. (b) $\epsilon_{1}=0.05, L_{1}=0.36$; bifurcation parameter $\epsilon_{2}$. (c) $\epsilon_{1}=0.54$, and period-two drive $L_{1}=0.77, L_{2}=0.22$; bifurcation parameter $\epsilon_{2}$. (d) $\epsilon_{2}=0.065 ; L_{1}=0.08$; bifurcation parameter is $\epsilon_{1}$. 
(a)
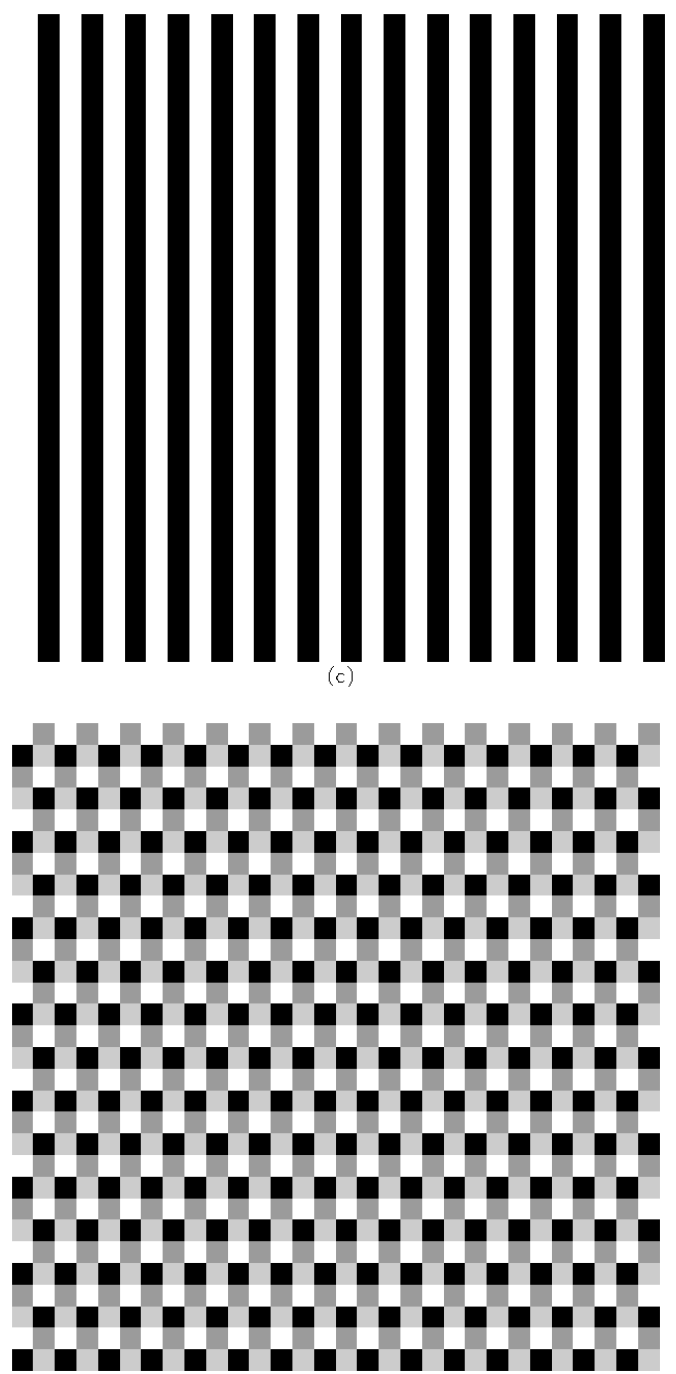

(b)
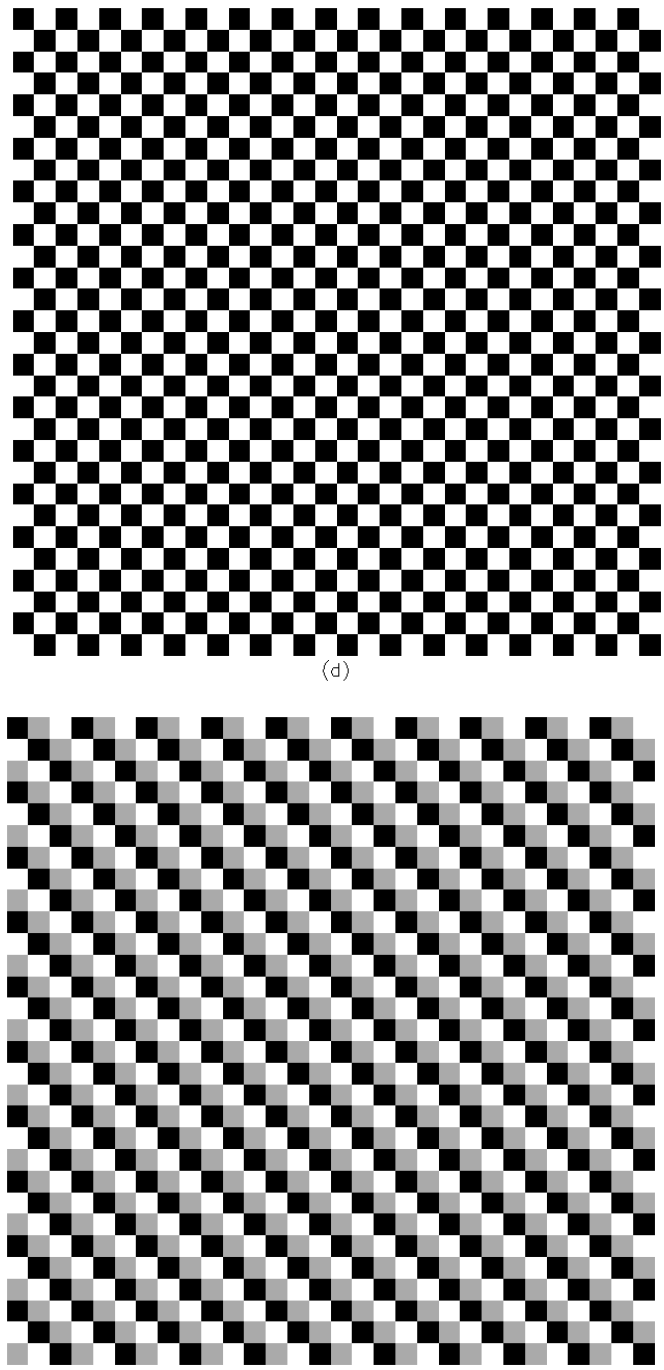

FIG. 2: Spatiotemporal patterns on a grey scale in the autonomous system (Eq11) with global mean field interaction $H$, at parameter values predicted from the analogy with the driven lattice in Fig. (). Size $N=30$; spatial index of $x_{t}^{i}$ runs horizontally and time runs from bottom to top. (a) Pattern $S(2,1) ; r=2, \epsilon_{1}=0.54, \epsilon_{2}=0.56$; constant $H=0.39$. (b) Pattern $S(2,2)$; $r=2, \epsilon_{1}=0.05, \epsilon_{2}=0.2$; constant $H=0.36$. (c) Pattern $S(2,4) ; r=2, \epsilon_{1}=0.54, \epsilon_{2}=0.51 ; H$ oscillates periodically between the values 0.77 and 0.22 . (d) Pattern $S(3,3) ; r=2, \epsilon_{1}=0.001, \epsilon_{2}=0.065$; constant $H=0.08$. 


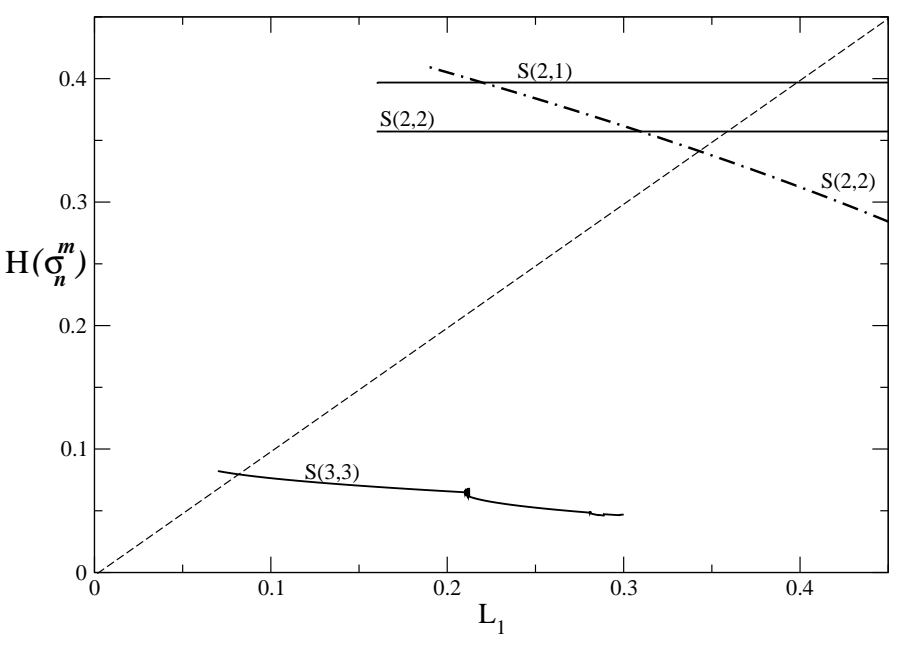

FIG. 3: Global coupling functions $H\left(\sigma_{n}^{m}\right)$ (r.h.s of Eq. (13)) associated to patterns $S(k, p)$ with constant drive, as a function of $L_{1}$. Continuous curves correspond to mean field global coupling with the parameters of Fig. (2) in each case. Dash-dotted curve displays a geometric mean global coupling function associated to the pattern $S(2,2)$ with parameters $r=2, \epsilon_{1}=0.005, \epsilon_{2}=0.4$; the intersection occurs at $L_{1}^{*}=0.3418$. 